Agricultural Economics Research Review

Vol. 25 (Conference Number) 2012 pp 473-483

\title{
Structure of Indebtedness of Households in Semi-Arid Tropics of India
}

\author{
A. Amarender Reddy \\ International Crops Research Institute for Semi-Arid Tropics (ICRISAT), \\ Patancheru-502 324, Andhra Pradesh
}

\begin{abstract}
The credit delivery system in India comprises both formal and informal institutions. The formal system comprises commercial banks, regional rural banks and cooperatives, while the predominant informal sources of credit are commission agents, traders, friends and relatives, chit funds and more recently, selfhelp groups. This paper has examined aspects like (i) who gets cheaper and who gets costly loans? (ii) how different borrowers and lenders are matched to each other? (iii) for what purpose households borrow (production, consumption, investment, social)? and lastly, (iv) are informal sources exploitative? The paper has used Village Dynamic Studies in South Asia data for 18 Semi-Arid Tropics villages in India comprising 857 households for the year 2009. The study has found that informal borrowings from relatives, friends, traders and commission agents continue to form a major source of total borrowings in the rural India. Generally, borrowings from formal sources are for large amounts at a lower interest rate compared to from informal borrowings. However, the majority of formal borrowings are skewed towards large landholders, and upper caste households who can offer collateral securities and also benefit from crop loans, for which basic eligibility criteria for getting loans is land. The borrowings from informal sources have been found distributed across all class and caste groups uniformly as these are mostly inter-personal borrowings with no collateral securities. About half of the borrowings from friends and traders carry no interest rate, but for very small amounts and for a shorter duration. However, on average informal sources have been found charging three-times interest rates that of formal sources if we account for productinput-credit market linkages.
\end{abstract}

Key words: Rural finance, interest rates, indebtedness, India

\section{JEL Classification: G2}

\section{Introduction}

In 2003, about 48.6 per cent of rural households were indebted in India. Households in the states falling in the Semi-Arid Tropics (SAT) have a higher level of indebtedness, the highest being in Andhra Pradesh (82.0\%), followed by Karnataka (61.6\%), Maharashtra (54.8\%), Gujarat (51.9\%) and Madhya Pradesh $(50.8 \%)$. The average size of loan was also highest in Andhra Pradesh (₹ 23965), followed by Karnataka

\footnotetext{
* Author for correspondence,

Email: a.amarenderreddy@cgiar.org
}

(₹ 18135), Maharashtra (₹ 16973), Gujarat (₹ 15526) and Madhya Pradesh (₹ 14218), when compared to all-India level of 12585 (NSSO, 2003). Borrowing from formal financial sources in many ways is not exploitative, but, from informal sources, it is exploitative (Binswanger and Sillers, 2002). Hence, banking sector in India has been attempting to limit most forms of informal finance by regulating them, banning them and allowing certain types of microfinance institutions. The latter policy aims to increase the availability of credit to low-income households and eliminate their reliance on usurious 
financing. Nonetheless, informal sector continues to dominate in the rural credit market (Tsai, 2004). The expert group feels that the objectives would be served better if farmers, especially small and marginal farmers, are organized through collectives like self-help groups (SHGs) and cooperatives (GoI, 2007; Reddy, 2006). Besides credit delivery, these collectives and formal institutional networks are expected to help the farmers in improving their farming practices through better accessing of appropriate technology, extension services, improved processing and marketing capabilities and risk management. However, the facts show that even after many financial reforms from both government and Reserve Bank of India in the past few decades, efforts to replace the informal financial sector with formal sector either through RRBs, cooperatives or even linking villagers with SHGs have not been fruitful. Obviously, there are some comparative ज. advantages, that the informal financial service ¿ providers (like relatives, friends, traders, commission

agents, input dealers, etc.) have in the rural areas like timely supply of credit, no procedural delays, mutual c understanding, symmetric and complete information between borrower and lender through daily social interactions in different markets (like credit-labour골 commodity markets).

The analysis of the coexistence of formal and E informal credit providers for such a long period with o their own competitive advantages like the former with o lower interest rates (about 7 - 9\% per annum) and the o later with convenience but with higher interest rates (24-36\% per annum) is the key challenge of theoretical and empirical work to provide a framework for understanding the rural credit markets in India. Why are there such higher rates of interest for at least some borrowers? How do large differences persist among interest rates of different borrowers? Why is there such a diversity of contract forms and intermediary structures? and why do such highly variable interest rates persist in equilibrium across borrowers? Keeping these broader theoretical and empirical questions, the paper addresses the following specific objectives: (i) who gets cheaper loans and who gets costly loans? (ii) how are different borrowers and lenders matched to each other? (iii) why households are borrowing (production, consumption, investment, social needs)?, and lastly (iv) are informal sources exploitative?

\section{Data and Methodology}

The data used in this paper were obtained from a larger research project entitled "Village Dynamic
Studies in South Asia (VDSA), in which ICRISAT research team collected a range of data from households of 18 selected villages from SAT India for the year 2009. The 18 villages in the VDSA studies of ICRISAT were selected from five states (Andhra Pradesh, Maharashtra, Madhya Pradesh, Gujarat and Karnataka), which represent the broad agro-climatic sub-regions in the semi-arid tropics of India. The selected villages were: Aurepalle, Dokur, JC Agraharam and Pamidipadu from Andhra Pradesh; Babrol, Karamdi Chingaria, Chatha, Makhiyala from Gujarat; Belladamadugu, Kappanimargi, Markabhinahalli, Tharati from Karnataka; Shirapur, Kalman, Kanzara, Kinkheda from Maharastra; and Papda and Rampur Kalan from Madhya Pradesh. The descriptive statistics and logistic regression models (step-wise regression) were used to find the extent of formal and informal borrowings among different sections of society and factors influencing the formal and informal borrowings.

\section{Results and Discussion}

In the ICRISAT sample data for the year 2009, out of 857 households, about 79 per cent were borrowers, and 21 per cent were non-borrowers from all sources (Table 1). It was important to see that about 51 per cent of households had not borrowed from the formal sector and only 36 per cent households had not borrowed from the informal sources. It indicated that still the majority of households borrow from the informal sources compared to formal sources in SATIndia (Table 2). About 46 per cent of total households had taken multiple loans - 13 per cent from formal sources and 26 per cent from informal sources and 7 per cent from both formal and informal sources. It was found that the share of upper caste and large landholders was higher in multiple loans from formal sources, while small and medium farmers had more multiple loans from informal sources. About 52 per cent of large landholders had taken multiple loans -29 per cent from formal sources, 19 per cent from informal sources and 4 per cent from both formal and informal sources. It also indicated that the large landholders as well as upper caste households were able to get multiple loans from formal sources as they had more land to show as collateral and had significant influence on formal sources of credit. Multiple loans from the formal sources were less in the case of landless, scheduled 
Table 1. Percentage of households with multiple loans from all sources in SAT-India: 2009

\begin{tabular}{|c|c|c|c|c|c|c|c|c|c|c|}
\hline \multirow{2}{*}{$\begin{array}{l}\text { Household } \\
\text { group }\end{array}$} & \multirow{2}{*}{$\begin{array}{c}\text { Non- } \\
\text { borrowers } \\
(\%)\end{array}$} & \multicolumn{6}{|c|}{ Number of times borrowed } & \multirow{2}{*}{$\begin{array}{c}\text { Total } \\
\text { borrowers }\end{array}$} & \multirow{2}{*}{$\begin{array}{c}\text { Borrowers } \\
\text { with multiple } \\
\text { loans (\%) }\end{array}$} & \multirow[t]{2}{*}{ Total } \\
\hline & & One & Two & Three & Four & Five & Six & & & \\
\hline Landless & 33 & 38 & 19 & 7 & 2 & 2 & 0 & 68 & 29 & 100 \\
\hline Small & 17 & 29 & 27 & 18 & 7 & 0 & 0 & 81 & 54 & 100 \\
\hline Medium & 20 & 35 & 22 & 12 & 9 & 1 & 0 & 79 & 45 & 100 \\
\hline Large & 16 & 32 & 26 & 12 & 9 & 4 & 0 & 83 & 52 & 100 \\
\hline $\mathrm{OBC}$ & 21 & 40 & 22 & 10 & 6 & 2 & 0 & 80 & 39 & 100 \\
\hline ST & 29 & 23 & 21 & 17 & 9 & 0 & 1 & 71 & 48 & 100 \\
\hline $\mathrm{SC}$ & 23 & 39 & 22 & 8 & 6 & 2 & 0 & 77 & 38 & 100 \\
\hline Others & 15 & 22 & 31 & 19 & 9 & 3 & 1 & 85 & 63 & 100 \\
\hline All & 21 & 33 & 24 & 13 & 7 & 2 & 0 & 79 & 46 & 100 \\
\hline total sample & 179 & 283 & 202 & 110 & 59 & 15 & 2 & 671 & 388 & 850 \\
\hline
\end{tabular}

Table 2. Percentage of households with multiple loans from formal and informal sources in SAT-India: 2009

\begin{tabular}{|c|c|c|c|c|c|c|c|c|c|c|c|c|c|}
\hline \multirow{3}{*}{$\begin{array}{l}\text { Household } \\
\text { group }\end{array}$} & \multicolumn{6}{|c|}{ Formal sources } & \multicolumn{6}{|c|}{ Informal sources } & \multirow[t]{3}{*}{ Total } \\
\hline & \multirow{2}{*}{$\begin{array}{c}\text { Non- } \\
\text { borrowers }\end{array}$} & \multicolumn{4}{|c|}{ Number of times borrowed } & \multirow{2}{*}{$\begin{array}{l}\text { Multiple } \\
\text { borrowers }\end{array}$} & \multirow{2}{*}{$\begin{array}{c}\text { Non- } \\
\text { borrowers }\end{array}$} & \multicolumn{4}{|c|}{ Number of times borrowed } & \multirow{2}{*}{$\begin{array}{l}\text { Multiple } \\
\text { borrowers }\end{array}$} & \\
\hline & & One & two & Three & Four & & & One & two & Three & Four & & \\
\hline Landless & 75 & 22 & 3 & 0 & 0 & 3 & 43 & 38 & 13 & 5 & 1 & 19 & 100 \\
\hline Small & 50 & 41 & 8 & 1 & 0 & 9 & 28 & 37 & 31 & 4 & 0 & 35 & 100 \\
\hline Medium & 48 & 40 & 11 & 1 & 0 & 12 & 36 & 39 & 18 & 5 & 1 & 24 & 100 \\
\hline Large & 35 & 37 & 26 & 2 & 1 & 29 & 43 & 38 & 12 & 6 & 1 & 19 & 100 \\
\hline $\mathrm{OBC}$ & 51 & 36 & 12 & 0 & 0 & 12 & 39 & 44 & 14 & 3 & 0 & 17 & 100 \\
\hline ST & 60 & 32 & 9 & 0 & 0 & 9 & 35 & 30 & 28 & 6 & 1 & 35 & 100 \\
\hline $\mathrm{SC}$ & 65 & 29 & 5 & 1 & 0 & 6 & 35 & 40 & 16 & 7 & 2 & 25 & 100 \\
\hline Others & 40 & 42 & 16 & 2 & 0 & 18 & 33 & 28 & 31 & 6 & 2 & 39 & 100 \\
\hline All & 51 & 36 & 12 & 1 & 0 & 13 & 36 & 38 & 20 & 5 & 1 & 26 & 100 \\
\hline Total sample & 437 & 306 & 99 & 7 & 1 & 107 & 310 & 324 & 168 & 41 & 7 & 216 & 850 \\
\hline
\end{tabular}

tribes and scheduled castes, hence they had to invariably depend on the informal sector as they were not able to provide the necessary collateral security to get loans from the formal sources.

Most of the borrowers had taken loans from the informal sources like relatives, friends, traders, commission agents, etc., but the borrowed amount was small and interest rates were high (Table 3 ). While the amount borrowed from the formal sources (commercial banks, RRBs and cooperative) was large and with lower interest rates. The average interest rates for borrowings from formal sources were lower - commercial banks, 9 per cent; cooperatives and RRBs, 7 per cent; as against 15 per cent from SHGs, 16 per cent from relatives and friends, 21 per cent from traders and commission agents, 20 per cent from mutual funds, 25 per cent from landlords, 20 per cent from others, and 5 per cent from employers. The average amount borrowed was highest from commercial banks (₹ 105298) for the duration of 20 months, followed by from RRBs (₹ 46893) for 15 months, chit funds (₹ 45142) for 19 months, landlords ( $₹$ 39917) for 17 months, cooperatives ( $₹ 27131$ ) for 14 months, employers (₹ 24765) for 7 months, from relatives (₹ 22921) for 12 months, from traders (₹ 16360) for 8 months and the least was from SHGs (₹ 9031) for 10 months. Overall, it was revealed that the borrowing from formal sources was for large amounts with longer 
Table 3. Sources of finance, amount, duration and interest rate

\begin{tabular}{|c|c|c|c|c|}
\hline Source & Description & $\begin{array}{l}\text { Average } \\
\text { amount } \\
()\end{array}$ & $\begin{array}{l}\text { Duration } \\
\text { (months) }\end{array}$ & $\begin{array}{l}\text { Interest rate } \\
\text { per annum } \\
(\%)\end{array}$ \\
\hline \multicolumn{5}{|c|}{ Formal sources } \\
\hline Commercial banks & $\begin{array}{l}\text { Borrowings from private, public and MNC banks } \\
\text { operating in India }\end{array}$ & 105298 & 20 & 9 \\
\hline RRBs & $\begin{array}{l}\text { Borrowings from Grameena Banks functioning under } \\
\text { the Regional Rural Banks Act, } 1976\end{array}$ & 46893 & 15 & 7 \\
\hline Cooperatives & Borrowings from cooperative banks & 27131 & 14 & 7 \\
\hline \multicolumn{5}{|c|}{ Informal sources } \\
\hline SHGs & $\begin{array}{l}\text { Borrowings from self-help groups promoted by NGOs, } \\
\text { banks, government. As they are mostly linked to banking } \\
\text { system or MFIs, it is more appropriate to call them as } \\
\text { "semi-formal sources". }\end{array}$ & 9031 & 10 & 15 \\
\hline Relatives & $\begin{array}{l}\text { Interpersonal borrowings - borrowings among friends, } \\
\text { relatives, neighbours or colleagues. Financial authorities } \\
\text { do not interfere with casual, interest-free lending }\end{array}$ & 22921 & 12 & 16 \\
\hline Traders & $\begin{array}{l}\text { Merchandise borrowings from traders, commission } \\
\text { agents, shopkeepers, input dealers to villagers } \\
\text { (which include trade credit, forward sales) }\end{array}$ & 16360 & 8 & 21 \\
\hline Chit funds & $\begin{array}{l}\text { Borrowings from indigenously organized savings and } \\
\text { credit groups, registered as companies, partnerships } \\
\text { and sole proprietorships }\end{array}$ & 45142 & 19 & 20 \\
\hline Landlords & Borrowings from landlords & 39917 & 17 & 25 \\
\hline
\end{tabular}

durations and also at lower interest rate as compared to from informal sources.

Most of the borrowers had used a major proportion of their borrowings for productive purposes, especially those who had borrowed from the formal sources (Table 4). Out of total households, 42 per cent had borrowed from relatives and friends, 32 per cent had borrowed from traders and commission agents, 22 per cent had borrowed from cooperatives, 15 per cent had borrowed from commercial banks, 8 per cent had borrowed from RRBs and the remaining 11 per cent had borrowed from other sources like chit funds, employer, etc. While households which had borrowed from informal sources (relatives, friends, SHGs and traders) had also spent a considerable amount on consumption purpose, some borrowers from relatives and friends also spent on social purpose. All together, agriculture followed by consumption were the major purposes for which households borrowed money.

It was interesting to see that more than half of the borrowers from relatives and friends had borrowed at zero rate of interest; and the same was applicable to borrowers from traders and commission agents (Table 5). However, field-level observations showed that there was prevalence of agricultural produce-input supplylabour-credit market linkages in most of the villages; hence if we take into account these linkages, the interest rates from traders and commission agents were higher than of commercial banks. It was also confirmed by the high interest rates charged by the relatives and traders, averaging about 32 per cent and 43 per cent, respectively for the remaining half of the borrowers. These figures also show that at least for some borrowers, the interest rates charged by relatives and traders were not exploitative and were convenient for both borrowers and lenders for small borrowings for duration of up to 8 months. About 60 per cent of borrowers from SHGs borrowed at below 12 per cent interest and 40 per cent at above 12 per cent interest, indicating that the SHGs linked with formal sources lend at lower interest rates.

The borrowings from commercial banks were for large amounts mainly for agricultural purposes like 
Table 4. Sources of credit and purpose for taking a loan

(\% of total households)

\begin{tabular}{|c|c|c|c|c|c|c|c|c|c|}
\hline Broad purpose & Loan utilization & Relatives & Traders & Cooperatives & SHGs & Banks & RRBs & Others & $\begin{array}{c}\% \text { of } \\
\text { households }\end{array}$ \\
\hline \multirow{3}{*}{$\begin{array}{l}\text { Production } \\
\text { /investment }\end{array}$} & Agriculture & 11 & 7 & 21 & 4 & 7 & 7 & 2 & 59 \\
\hline & Starting business & 1 & 1 & 0 & 1 & 1 & 0 & 0 & 4 \\
\hline & Purchasing of livestock & 1 & 0 & 0 & 1 & 0 & 0 & 0 & 2 \\
\hline \multirow[t]{3}{*}{ Consumption } & Consumption & 10 & 19 & 0 & 7 & 0 & 0 & 4 & 40 \\
\hline & Medical care & 4 & 2 & 0 & 0 & 0 & 0 & 0 & 6 \\
\hline & Education & 2 & 1 & 0 & 1 & 0 & 0 & 0 & 4 \\
\hline \multirow[t]{4}{*}{ Social } & Social functions & 2 & 0 & 0 & 0 & 0 & 0 & 1 & 5 \\
\hline & Other obligations & 11 & 3 & 1 & 2 & 3 & 0 & 4 & 24 \\
\hline & $\begin{array}{l}\text { Borrower } \\
\text { households }\end{array}$ & 42 & 32 & 22 & 15 & 12 & 8 & 11 & 80 \\
\hline & $\begin{array}{l}\text { Non-borrower } \\
\text { households }\end{array}$ & 58 & 68 & 78 & 85 & 88 & 92 & 97 & 20 \\
\hline
\end{tabular}

Note: Others include employers, chit funds and landlords

Table 5. Source of credit amount, interest and duration

\begin{tabular}{|c|c|c|c|c|c|c|c|c|}
\hline \multirow{2}{*}{$\begin{array}{l}\text { Source of } \\
\text { credit }\end{array}$} & \multirow{2}{*}{$\begin{array}{l}\text { Amount } \\
\text { (₹) }\end{array}$} & \multirow{2}{*}{$\begin{array}{l}\text { Duration } \\
\text { (months) }\end{array}$} & \multirow{2}{*}{$\begin{array}{c}\text { Number of } \\
\text { samples }\end{array}$} & \multicolumn{5}{|c|}{ Interest (\%) per annum } \\
\hline & & & & Minimum & Maximum & Mean & $\begin{array}{l}\text { Standard } \\
\text { deviation }\end{array}$ & $\begin{array}{l}\mathrm{CV} \\
(\%)\end{array}$ \\
\hline Relatives (Interest $=0$ ) & 11780 & 8 & 205 & & & & & \\
\hline Relatives (Interest $>0$ ) & 33962 & 15 & 201 & 2 & 72 & 32 & 11 & 34 \\
\hline Traders $($ Interest $=0)$ & 3478 & 4 & 157 & & & & & \\
\hline Traders (Interest $>0$ ) & 29826 & 7 & 149 & 9 & 120 & 43 & 19 & 44 \\
\hline Cooperatives & 27131 & 14 & 199 & 3 & 24 & 7 & 4 & 52 \\
\hline SHGs & 9031 & 10 & 145 & 3 & 36 & 15 & 10 & 64 \\
\hline Commercial banks & 105298 & 20 & 114 & 3 & 24 & 9 & 4 & 49 \\
\hline RRBs & 46893 & 15 & 71 & 2 & 18 & 7 & 4 & 49 \\
\hline Employers & 17959 & 6 & 31 & 0 & 36 & 5 & 11 & 222 \\
\hline Others & 15236 & 13 & 26 & 0 & 36 & 20 & 8 & 38 \\
\hline Landlords & 31542 & 18 & 24 & 0 & 36 & 25 & 11 & 42 \\
\hline Chit funds & 39917 & 17 & 19 & 12 & 36 & 25 & 9 & 45 \\
\hline
\end{tabular}

drilling of bore-wells and purchasing of farm implements, land and livestock and were for a longer duration, up to 144 months (Table 6). The loan amount for consumption purpose was small and was for a shorter duration, 12-34 months. Next to commercial banks, the average amount borrowed was high from cooperatives. A major share of borrowings from the cooperatives was used for consumption and social purposes, while the borrowings from RRBs were almost entirely used for agricultural purpose and very few were for other purposes.
In general, borrowings of large amounts were maximum for agriculture, followed by social purpose and the least for consumption purpose from informal sources (Table 7). The average amount of borrowings from relatives was larger than from traders and the smallest in the case of borrowings from SHGs. Among all the borrowings, purchase of farm implements and drilling of bore-wells involved a larger amount and long period of repayment. Under the category of consumption, borrowings were more for education, followed by medical purpose. 
Reddy : Structure of Indebtedness of Households in Semi-Arid Tropics of India

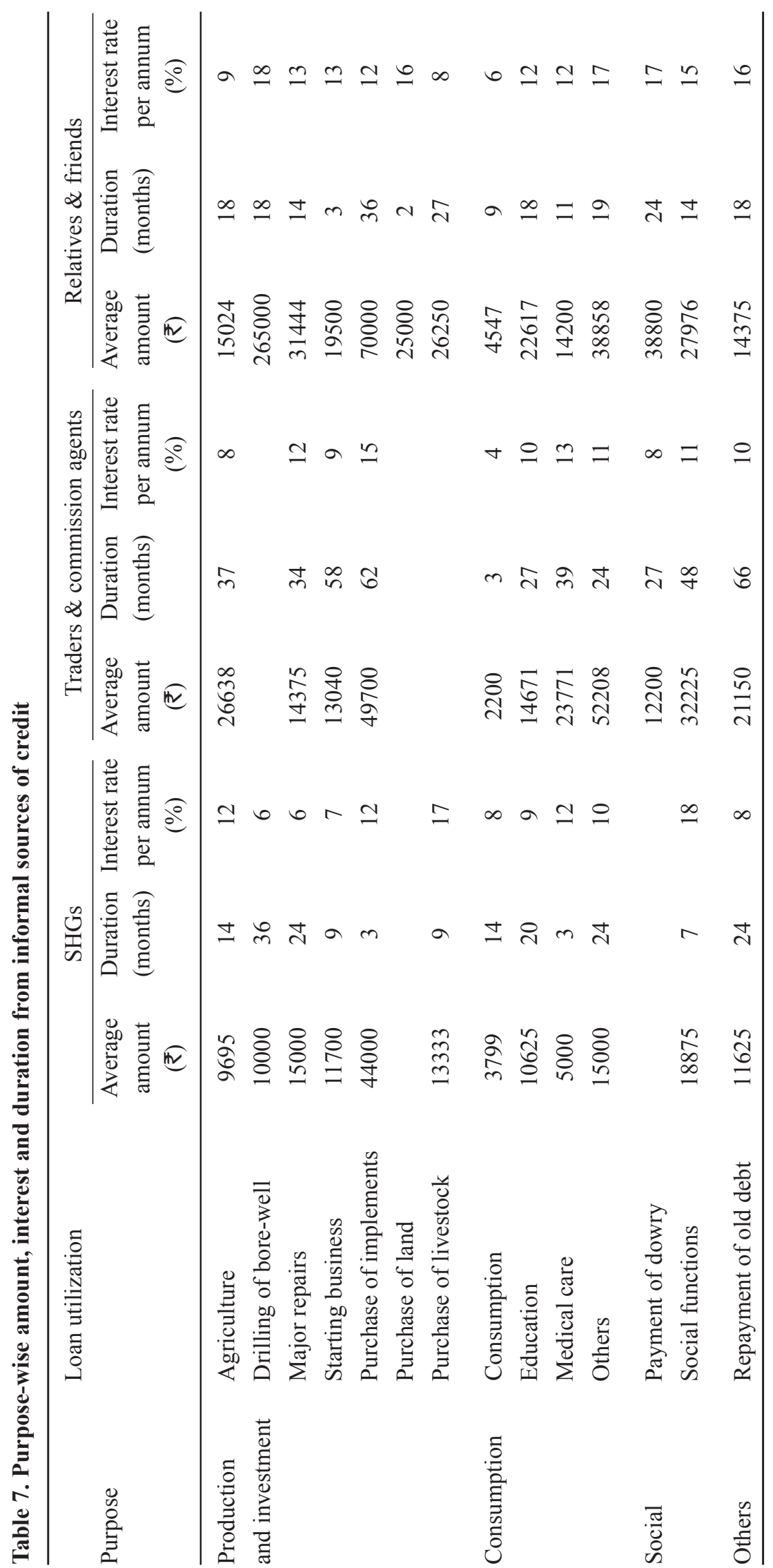


It is interesting to see that large landholders, caste households could influence the banking officials forward caste households and higher-educated households borrowed large amounts from formal sources compared with their counterparts (Table 8). It was also seen that the large landholders and forwardto get a higher amount of loan as it is mostly at a lower interest rates and many a times it is waived off due to political, economic and other considerations. It is also true that most of the large landholders do cultivate large

Table 8. Borrowings from formal sources by social group

\begin{tabular}{|c|c|c|c|c|c|c|c|c|c|c|}
\hline \multirow[b]{2}{*}{ Item } & \multirow[t]{2}{*}{ Category } & \multicolumn{3}{|c|}{ Commercial banks } & \multicolumn{3}{|c|}{ RRBs } & \multicolumn{3}{|c|}{ Cooperatives } \\
\hline & & $\begin{array}{l}\text { Average } \\
\text { amount } \\
\text { (₹) }\end{array}$ & $\begin{array}{l}\text { Duration } \\
\text { (months) }\end{array}$ & $\begin{array}{c}\text { Interest } \\
\text { rate per } \\
\text { annum } \\
(\%)\end{array}$ & $\begin{array}{c}\text { Average } \\
\text { amount } \\
\text { (₹) }\end{array}$ & $\begin{array}{l}\text { Duration } \\
\text { (months) }\end{array}$ & $\begin{array}{l}\text { Interest } \\
\text { rate per } \\
\text { annum } \\
(\%)\end{array}$ & $\begin{array}{c}\text { Average } \\
\text { amount } \\
\text { (₹) }\end{array}$ & $\begin{array}{l}\text { Duration } \\
\text { (months) }\end{array}$ & $\begin{array}{c}\text { Interest } \\
\text { rate per } \\
\text { annum } \\
(\%)\end{array}$ \\
\hline \multirow[t]{3}{*}{ Farm-size } & Landless & 52083 & 10 & 30 & 49208 & 11 & 27 & 21375 & 7 & 14 \\
\hline & Small & 58252 & 10 & 18 & 35033 & 9 & 12 & 21898 & 8 & 12 \\
\hline & Medium & 71922 & 9 & 18 & 39107 & 8 & 13 & 31668 & 8 & 15 \\
\hline & Large & 160955 & 8 & 14 & 56604 & 8 & 11 & 37757 & 7 & 13 \\
\hline \multirow[t]{4}{*}{ Caste } & $\mathrm{OBC}$ & 133801 & 8 & 15 & 46690 & 8 & 13 & 24151 & 7 & 15 \\
\hline & $\mathrm{SC}$ & 55873 & 10 & 26 & 26417 & 11 & 12 & 17495 & 9 & 10 \\
\hline & $\mathrm{ST}$ & 72167 & 8 & 14 & 20000 & 6 & 12 & 31872 & 8 & 14 \\
\hline & Others & 100813 & 9 & 19 & 56274 & 10 & 13 & 34091 & 7 & 12 \\
\hline \multirow[t]{3}{*}{ Religion } & Hindu & 113058 & 9 & 16 & 47606 & 9 & 13 & 28810 & 8 & 14 \\
\hline & Muslim & 35467 & 6 & 38 & 32000 & 5 & 12 & 27883 & 6 & 12 \\
\hline & Others & 47500 & 11 & 27 & - & - & - & 14817 & 11 & 7 \\
\hline \multirow{6}{*}{$\begin{array}{l}\text { Educational } \\
\text { attainment of } \\
\text { household- } \\
\text { head }\end{array}$} & Illiterate & 46198 & 9 & 16 & 38872 & 8 & 9 & 24217 & 7 & 11 \\
\hline & Primary & 56932 & 8 & 17 & 50466 & 8 & 11 & 31689 & 8 & 14 \\
\hline & Middle & 78778 & 10 & 30 & 46883 & 9 & 12 & 22806 & 8 & 17 \\
\hline & Inter $(10+2)$ & 66369 & 10 & 19 & 42007 & 8 & 20 & 47240 & 8 & 14 \\
\hline & Higher middle & 190746 & 9 & 15 & 54283 & 9 & 17 & 31995 & 8 & 13 \\
\hline & Higher educated & 214785 & 9 & 15 & 78333 & 9 & 11 & 26086 & 7 & 12 \\
\hline \multirow{5}{*}{$\begin{array}{l}\text { Age group } \\
\text { (years) }\end{array}$} & Young (below 25) & 34000 & 10 & 6 & 43500 & 7 & 12 & 10700 & 11 & 7 \\
\hline & $\begin{array}{l}\text { Middle-aged } \\
(26-35)\end{array}$ & 289090 & 8 & 16 & 53821 & 8 & 17 & 26684 & 7 & 12 \\
\hline & $\begin{array}{l}\text { Middle-aged } \\
(36-45)\end{array}$ & 86568 & 9 & 16 & 53054 & 9 & 10 & 22336 & 8 & 12 \\
\hline & Aged (45-60) & 77160 & 9 & 18 & 42980 & 9 & 13 & 31227 & 8 & 15 \\
\hline & Old $(>60)$ & 55047 & 9 & 18 & 45833 & 9 & 13 & 34991 & 7 & 13 \\
\hline \multirow{9}{*}{$\begin{array}{l}\text { Main } \\
\text { occupation } \\
\text { of households }\end{array}$} & Farming & 131027 & 9 & 16 & 47470 & 9 & 13 & 30577 & 8 & 14 \\
\hline & Farm labour & 33754 & 8 & 34 & 17000 & 8 & 11 & 15833 & 7 & 11 \\
\hline & Livestock & 71500 & 11 & 11 & 15000 & 12 & 24 & 11117 & 7 & 10 \\
\hline & Caste occupation & 29100 & 8 & 8 & 23000 & 8 & 6 & 19450 & 5 & 9 \\
\hline & Non-farm labour & 19417 & 11 & 14 & 34500 & 8 & 6 & 23333 & 7 & 13 \\
\hline & Business + Others & 50400 & 11 & 16 & 83500 & 10 & 28 & 24844 & 8 & 11 \\
\hline & Salaried & 127111 & 11 & 14 & 20000 & 12 & 6 & 51232 & 9 & 17 \\
\hline & Domestic work & 23333 & 8 & 13 & 18500 & 5 & 8 & 9813 & 8 & 12 \\
\hline & No work & 263125 & 10 & 53 & 41167 & 8 & 11 & 57200 & 8 & 18 \\
\hline
\end{tabular}


area which requires more working and fixed capital. Hence, under normal circumstances mere outstanding indebtedness with formal sector may not be a distress phenomenon in most of the cases but only indicates their requirement for carrying out productive activities.

While the villagers can take loan for any purpose from the informal sources (Table 9) and generally they don't require any collateral security. Informal borrowings are generally for smaller amounts and for a shorter duration but at higher interest rates. As is evident from Table 9, these are accessible to all households, irrespective of their landholding class, caste and educational level. However, it was interesting to see that there was no significant difference in the

Table 9. Borrowings from informal sources by social group

\begin{tabular}{|c|c|c|c|c|c|c|c|c|c|c|}
\hline \multirow[b]{2}{*}{ Item } & \multirow[t]{2}{*}{ Category } & \multicolumn{3}{|c|}{ SHGs } & \multicolumn{3}{|c|}{ Relatives \& friends } & \multicolumn{3}{|c|}{ Traders \& commission agents } \\
\hline & & $\begin{array}{c}\text { Average } \\
\text { amount } \\
\text { (₹) }\end{array}$ & $\begin{array}{l}\text { Duration } \\
\text { (months) }\end{array}$ & $\begin{array}{l}\text { Interest } \\
\text { rate per } \\
\text { annum } \\
(\%)\end{array}$ & $\begin{array}{l}\text { Average } \\
\text { amount } \\
\text { (₹) }\end{array}$ & $\begin{array}{l}\text { Duration } \\
\text { (months) }\end{array}$ & $\begin{array}{c}\text { Interest } \\
\text { rate per } \\
\text { annum } \\
(\%)\end{array}$ & $\begin{array}{c}\text { Average } \\
\text { amount } \\
\text { (₹) }\end{array}$ & $\begin{array}{l}\text { Duration } \\
\text { (months) }\end{array}$ & $\begin{array}{c}\text { Interest } \\
\text { rate per } \\
\text { annum } \\
(\%)\end{array}$ \\
\hline \multirow[t]{4}{*}{ Farm-size } & Landless & 9213 & 13 & 14 & 17326 & 16 & 12 & 10764 & 20 & 9 \\
\hline & Small & 9539 & 16 & 12 & 15810 & 9 & 9 & 6531 & 13 & 6 \\
\hline & Medium & 8857 & 15 & 12 & 31744 & 17 & 12 & 20455 & 24 & 8 \\
\hline & Large & 9108 & 17 & 10 & 48397 & 21 & 12 & 35393 & 29 & 10 \\
\hline \multirow[t]{4}{*}{ Caste } & $\mathrm{OBC}$ & 8759 & 16 & 12 & 33280 & 21 & 14 & 26778 & 31 & 10 \\
\hline & $\mathrm{SC}$ & 8377 & 12 & 11 & 19553 & 22 & 11 & 14074 & 31 & 9 \\
\hline & ST & 11829 & 22 & 10 & 12356 & 3 & 8 & 3788 & 7 & 5 \\
\hline & Others & 9897 & 15 & 12 & 22130 & 5 & 8 & 7615 & 7 & 5 \\
\hline \multirow[t]{3}{*}{ Religion } & Hindu & 9110 & 16 & 12 & 26055 & 15 & 11 & 15685 & 20 & 8 \\
\hline & Muslim & 18000 & 24 & 13 & 21823 & 8 & 8 & 7217 & 17 & 7 \\
\hline & Others & 8500 & 10 & 14 & 8715 & 12 & 14 & 14863 & 32 & 6 \\
\hline \multirow{5}{*}{$\begin{array}{l}\text { Educational } \\
\text { attainment of } \\
\text { household- } \\
\text { head }\end{array}$} & Illiterate & 7409 & 16 & 11 & 26612 & 19 & 11 & 16551 & 22 & 8 \\
\hline & Primary & 8179 & 16 & 11 & 17418 & 10 & 10 & 12163 & 16 & 7 \\
\hline & $10+2$ & 14469 & 21 & 10 & 27370 & 9 & 9 & 5930 & 13 & 6 \\
\hline & Higher middle & 10326 & 15 & 13 & 35087 & 13 & 13 & 18913 & 18 & 8 \\
\hline & Higher educated & 10875 & 13 & 11 & 25121 & 7 & 10 & 18260 & 15 & 8 \\
\hline \multirow[t]{5}{*}{$\begin{array}{l}\text { Age group } \\
\text { (years) }\end{array}$} & $\begin{array}{l}\text { Young } \\
\text { (below 25) }\end{array}$ & 9129 & 15 & 12 & 4000 & 0 & 12 & 3000 & 60 & 3 \\
\hline & $\begin{array}{l}\text { Middle-aged } \\
(26-35)\end{array}$ & 7474 & 18 & 11 & 28936 & 19 & 12 & 17069 & 26 & 9 \\
\hline & $\begin{array}{l}\text { Middle-aged } \\
(36-45)\end{array}$ & 9979 & 16 & 12 & 20452 & 12 & 10 & 15280 & 16 & 7 \\
\hline & Aged (45-60) & 8801 & 15 & 12 & 26120 & 14 & 11 & 16229 & 22 & 8 \\
\hline & Old $(>60)$ & 10151 & 14 & 13 & 30018 & 15 & 12 & 13585 & 17 & 7 \\
\hline \multirow{9}{*}{$\begin{array}{l}\text { Main } \\
\text { occupation } \\
\text { of households }\end{array}$} & Farming & 9052 & 16 & 12 & 33018 & 15 & 12 & 20113 & 24 & 8 \\
\hline & Farm labour & 4352 & 17 & 8 & 10030 & 11 & 9 & 8183 & 14 & 7 \\
\hline & Livestock & 9525 & 14 & 11 & 11974 & 12 & 10 & 7274 & 20 & 8 \\
\hline & Caste occupation & 8033 & 16 & 12 & 29990 & 31 & 12 & 7900 & 28 & 7 \\
\hline & Non-farm labour & 8250 & 14 & 15 & 22488 & 21 & 13 & 10513 & 17 & 8 \\
\hline & Business + others & 11752 & 14 & 13 & 25643 & 10 & 12 & 18801 & 16 & 9 \\
\hline & Salaried & 13889 & 15 & 14 & 18603 & 11 & 9 & 9043 & 16 & 7 \\
\hline & Domestic work & 7975 & 20 & 14 & 10771 & 11 & 8 & 6571 & 11 & 6 \\
\hline & No work & 23875 & 25 & 8 & 21186 & 10 & 11 & 9348 & 8 & 5 \\
\hline
\end{tabular}


Table 10. Factors influencing the formal and informal borrowings by households

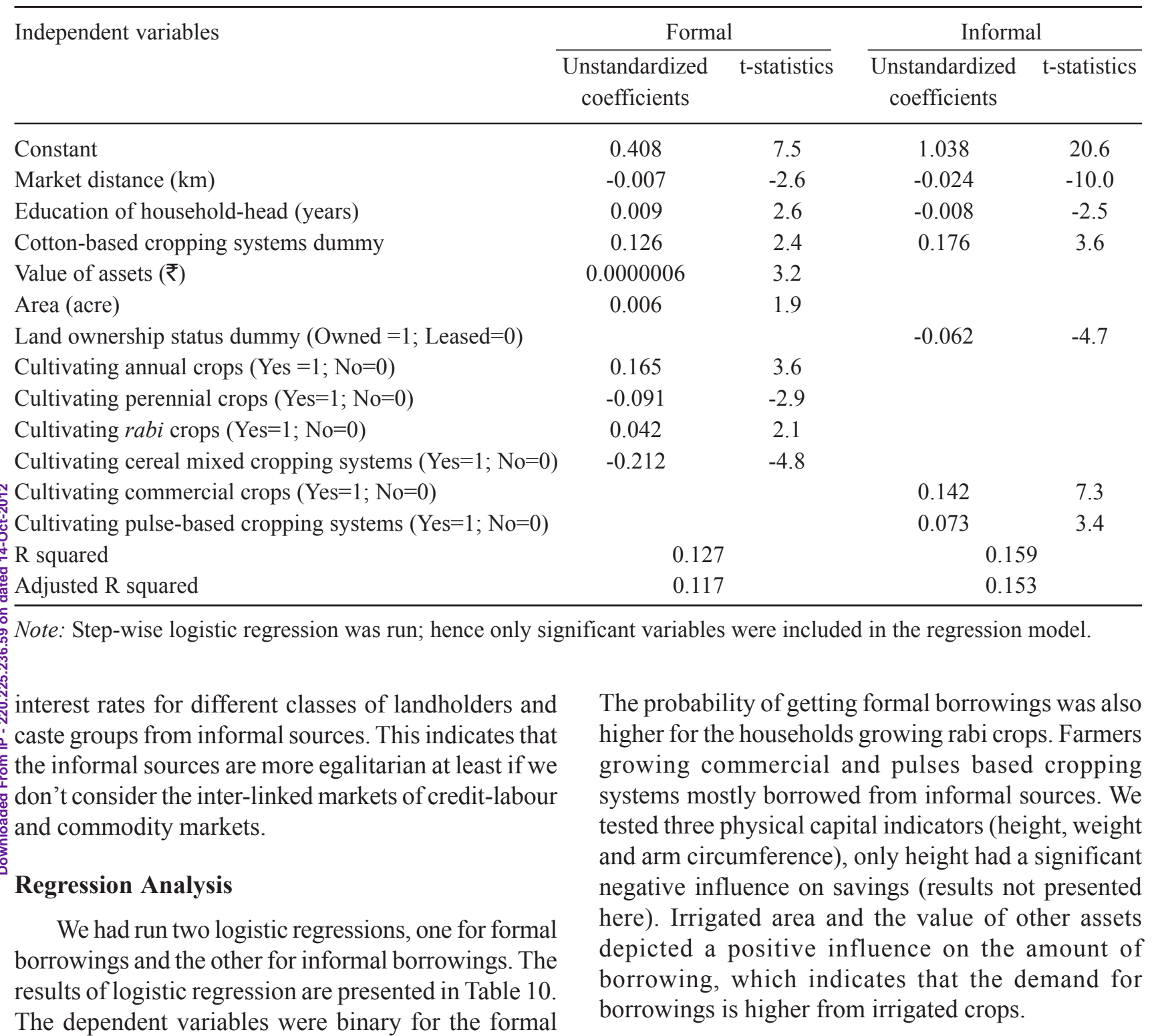
borrowing model (formal borrowing: $\mathrm{Yes}=1 ; \mathrm{No}=0$ ) and for informal borrowing (informal borrowing: Yes $=1 ; \mathrm{No}=0$ ). As expected, the years of education of household-head influenced the probability of borrowing positively from formal sources, and negatively from informal sources. The probability of borrowing from both formal and informal sources was seen higher among cotton-growing farmers. Market distance negatively influenced the probability of both formal and informal borrowings. Area and total assets had a positive influence on probability of formal borrowing, but had no influence on informal borrowing. Owning land depicted a negative influence on probability of borrowings from informal sources.

\section{Conclusions}

The study has revealed that as agriculture and rural areas are being exposed to more commercialization, the financial transactions of the rural households are increasing. More rural households are borrowing now than in earlier years. The indebtedness of the sample households has been higher compared to the indebtedness reported by NSSO in Situation Assessment Survey of Farmers in the SAT States. The majority of households borrow more from informal sources (like relatives, friends, traders and commission agents) than from formal sources of credit. The majority of households borrow for productive purposes like for 
agriculture, investment in bore-wells, purchase of livestock and cattle, etc, only a few take loans to meet their households' consumption and social needs.

The study has indicated that about 80 per cent of rural households have borrowed from either formal or informal sources and about 46 per cent of households have taken multiple loans. The relatives and traders have been found important players in credit field and they even supply credit at zero per cent interest for about 50 per cent of their customers. This shows the inter-linked credit-commodity-labour markets. It also indicates that it is a social necessity in the rural India, as they dominate in small loans for a shorter duration and with probably with a low transaction cost. However, the relatives and traders charges high interest rates for the another group of borrowers, which indicates they take the advantage of scarcity of credit in the rural areas from formal sources. The borrowings from formal sources are for larger amount of money with longer periods at lower interest rates, and are mostly favourable to large landholders, upper caste households, and irrigated land owners as they possess necessary collateral securities of land and other assets.

Cropping system has been found to play an important role in the sources of borrowings; for example, cotton farmers borrow more from both formal and informal sources. They also require more credit as their working and fixed capital needs are very high. These results have been confirmed with the regression analysis that the asset value, land area, and irrigated area are important demand-side factors. Regression results have also shown that interest rate of the formal sector is insignificant in households' borrowing decisions.

\section{Acknowledgements}

The paper is drawn from project "Study of Spatial and Temporal Dynamics of Labour Market Behaviour by using Household Panel Data of ICRISAT" under CGIAR Research Program-2 in year 2012. The authors are thankful to the constructive criticism of the Managing Editors of the journal.

\section{References}

Binswanger, H.P. and Sillers, D.A. (2002) Risk Aversion and credit constraints in farmers decision-making: A reinterpretation, Journal of Development Studies, 20: 5-21.

GoI (Government of India) (2007) Report of the Expert Group on Agricultural Indebtedness. Banking Division, Department of Economic Affairs, Ministry of Finance, New Delhi, July.

NSSO (National Sample Survey Organisation) (2003) Situation Assessment Survey of Farmers, Indebtedness of Farmer Households, NSS 59th Round, Report No 498, Ministry of Statistics and Programme Implementation, New Delhi.

Reddy, A.A. (2006) Productivity growth of regional rural banks, Economic and Political Weekly, 41(11): 10791086.

Tsai, K.S. (2004) Imperfect substitutes the local political economy of informal finance and microfinance in rural China and India, World Development, 32(9): 14871507. 